\title{
Pulmonary Venous Flow in Normal Dogs Recorded by Transthoracic Echocardiography: Techniques, Anatomic Validations and Flow Characteristics
}

\author{
Chiang-Hua CHIANG, Mitsuyoshi HAGIO*, Hiroko YOSHIDA and Shozo OKANO \\ Department of Small Animal Medicine, Faculty of Veterinary Medicine and Animal Sciences, Kitasato University, Towada, Aomori 034, \\ Japan
}

(Received 12 June 1997/Accepted 6 October 1997)

\begin{abstract}
To observe pulmonary venous flow in dogs, the echocardiographic imaging planes and the techniques for examination, and the validations of anatomic location were investigated. Then, the velocity pattern of pulmonary venous flow was recorded in normal conscious dogs. Six imaging planes were available for observing the right or left caudal lobe pulmonary venous flow with twodimensional or pulsed Doppler echocardiography. Of these, the left lateral apical 4-chamber view can be applied as standard view, because the pulmonary venous flow and transmitral flow could be recorded in this view simultaneously with small sampling angle. The velocity pattern of pulmonary venous flow demonstrated two forward waves in 19 of 20 dogs examined, with one peak occurring during ventricular systole and another during ventricular diastole. A reversed flow during atrial contraction was also seen in 11 dogs. In the two forward waves, the mean peak velocity and velocity-time integral of ventricular diastolic forward flow were significantly higher than those of systolic forward flow $(46.49 \pm 6.79$ vs. $31.13 \pm 4.92 \mathrm{~cm} / \mathrm{s}, \mathrm{p}<0.0001$ and $8.18 \pm 1.84$ vs. $5.14 \pm 0.82 \mathrm{~cm}, \mathrm{p}<0.0001$, respectively). The deceleration time of diastolic forward flow shortened with the increase of heart rate $(r=-0.87, p<0.0001)$. Pulmonary venous flow in dogs can be observed under transthoracic two-dimensional or pulsed Doppler echocardiography. — KEY wORDS: canine, pulmonary venous flow, pulsed Doppler, transthoracic echocardiography.
\end{abstract}

J. Vet. Med. Sci. 60(3): 333-339, 1998

The pulmonary vein transfuses blood to the left atrium from the lungs. In the past, the flow of blood through the pulmonary vein had been studied by two groups of investigators. One group emphasized the suction forces of the left atrium and ventricle $[4,11,12,16]$, while the other considered the propulsive force of the right ventricular contraction mainly [13]. In the previous studies, the pulsed Doppler echocardiographic velocity pattern of pulmonary venous flow (PVF) mirrored the wave-form of left atrial pressure where there are two peaks during y descent and $\mathrm{x}$ descent. Therefore, the hemodynamics of PVF seem to be influenced by the functions of left heart vis a fronte significantly [17].

Recently, pulsed Doppler echocardiographic assessment of PVF has become a widely accepted method with transesophageal probe to help evaluating left ventricular diastolic function in various cardiac diseases in human medicine $[9,14,18,20]$. However, transesophageal echocardiography can not be performed in dogs without anesthesia limiting its clinical utility. Although the investigations of PVF have been performed in human by pulsed Doppler echocardiography clinically [10] or in the dog experimental models [1,3,19], no study has been reported yet about the PVF for clinical utility in veterinary medicine.

This study, accordingly, was conducted to examine the feasibility of observing PVF with transthoracic pulsed Doppler echocardiography in dogs, and then, to develop the

* Correspondence to: Dr. Hagio, M., Department of Small Animal Medicine, Faculty of Veterinary Medicine and Animal Sciences, Kitasato University, Towada, Aomori 034, Japan. available imaging planes and the techniques for examination, and to confirm the anatomic location of pulmonary veins on the imaging plane, finally, to record the characters of the pulsed Doppler pattern of PVF in normal dogs.

\section{MATERIALS AND METHODS}

The available imaging planes and the anatomic validation in two-dimensional echocardiography: Five mongrel dogs weighing 8 to $11 \mathrm{~kg}$ (mean weight $9.30 \pm 1.52 \mathrm{~kg}, 3$ males and 2 females) obtained from an animal shelter were determined as healthy based on the results of routine physical examination and hematology. No evidence of cardiac or pulmonary abnormality was seen under either auscultation or two-dimensional echocardiography. These dogs were examined in the right or left lateral recumbency under consciousness with two-dimensional and color Doppler echocardiography by an ultrasound instrument (EUB-565A; HITACHI Medical Corporation) and a $5 \mathrm{MHz}$ sector scanning probe (EUP-S32; HITACHI Medical Corporation). The presumed imaging planes for observing the simulacrums of the pulmonary vein were obtained. Then, all five dogs were prepared for lateral thoracotomy.

The anesthetic protocol included premedication with atropine $(0.02 \mathrm{mg} / \mathrm{kg}$ intramuscular $)$ and droperidol $(0.5 \mathrm{mg} /$ $\mathrm{kg}$ intravenous), induction with pentobarbital sodium (20 $\mathrm{mg} / \mathrm{kg}$ intravenous), and maintenance with Halothane (1\%$1.5 \%$ ). Thoracotomy was performed in two dogs from left lateral and in three dogs from right lateral at the fifth or sixth intercostal space after routine aseptic preparations. From right lateral, the caudal lobe ( 2 of 3 dogs) or middle lobe ( 1 of 3 dogs) pulmonary vein, and from left lateral, the 
caudal lobe pulmonary vein were certified. The PVF was determined beforehand by color Doppler ultrasound with a 7.5 MHz sector scanning probe (EUP-S33; HITACHI Medical Corporation) and by directly contacting the junction of the pulmonary vein and left atrium. Pulmonary veins were separated by blunt dissection near the junction, then two silk sutures were used to ligate the vein to about onehalf its original diameter. It was measured by color and pulsed Doppler ultrasound whether the color signal of the flow changes to mosaic jet signal or the flow velocity increases. After the mosaic jet signal or the increased flow velocities were certified, the thoracotomy incision was closed as usual. At postoperative fifth to seventh day, the five dogs were examined again, using same imaging planes that were found preoperatively, to identify the ligated pulmonary vein. All graphic imagines were recorded on a S-VHS videocassette recorder (SVO-9500MD; SONY) for retrospective analysis.

The velocity characters of PVF: After the available imaging plane has been obtained, twenty healthy mongrel dogs weighing 4 to $12 \mathrm{~kg}$ (mean weight $8.0 \pm 2.1 \mathrm{~kg}, 8$ males and 12 females) without evidence of cardiac abnormalities or Dirofilariae immitis infections were used for observing the normal velocity pattern of PVF. Their estimated ages were between 1 and 3 years old according to previous report [5]. Authors selected the most suitable imaging plane, left lateral apical 4-chamber view, to examine the flow pattern of right caudal lobe pulmonary vein. The PVF was recognized as red signal from the bottom in color Doppler mode. The sample volume of pulsed Doppler echocardiography was set just distal to the orifice of the vein, and the size of sample volume was set at $2 \mathrm{~mm}$. The sweep speed was set at the highest $(1.25 \mathrm{sec} /$ screen) of the ultrasound instrument. The transmitral flow (TMF) was recorded as usual with setting the sample volume at the tip level of mitral leaflets for comparing with PVF. Correction for the angle between Doppler sampling line and PVF or TMF was applied as needed. Recordings were obtained while the dogs were quiet without sedation.

Analysis of data: Flow velocity tracings were measured according to the schema demonstrated in Fig. 1 by using the software operating function in the ultrasound instrument. PVF tracing was measured by the moving of a track ball to determine the peak velocity of forward flow that occurred during ventricular systole (S wave) and diastole (D wave), and the peak velocity of reversed flow can also be seen at atrial contraction (AS wave). The velocity-time integrals (VTI) of S waves and D waves were measured by tracing the outer borders of the Doppler curves. When the D wave velocity began before the $S$ wave velocity had decreased to the zero baseline, the VTI of the S wave was defined as the area under the traced line from the onset of $S$ wave to the onset of D wave. The VTI of D wave was defined as the area from the onset to the end of the flow. The systolic fraction was expressed as VTI of S wave/(VTI of S wave+VTI of D wave). The peak velocity and VTI of transmitral early filling flow (E wave) and late filling flow

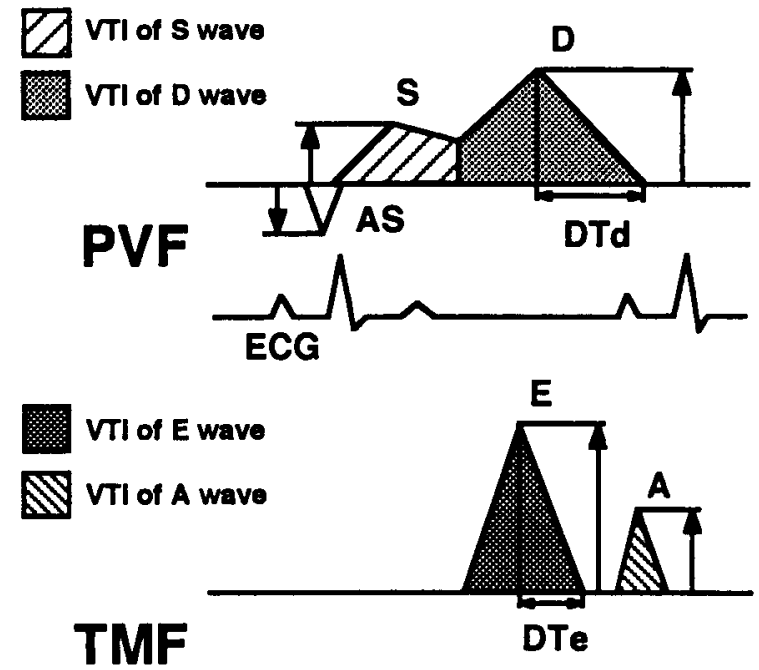

Fig. 1. Schema of pulsed Doppler echocardiographic pattern of pulmonary venous flow of dog and the measuring item. (S) the peak systolic forward flow velocity, (D) the peak diastolic forward flow velocity and (AS) the peak atrial systolic reversed flow velocity of PVF; (E) the peak early diastolic filling flow velocity and (A) the peak late diastolic filling flow velocity of TMF; (DTd) the deceleration time of diastolic forward flow of PVF; (DTe) the deceleration time of early diastolic filling flow of TMF.

(A wave) were measured respectively as usual. The deceleration time of $\mathrm{D}$ wave and $\mathrm{E}$ wave were measured from the peak of the velocity tracing to the point where the extrapolated line intersected the baseline.

Statistical analysis: Each variable was obtained by averaging three consecutive heart beats. All values are expressed as mean \pm SD. Statistical analysis was performed with a statistical software (StatView 4.02, Abacuus Concepts, Inc.) of a personal computer (Macintosh, Apple Japan, Inc.). Tests of correlation and significance were analyzed by Pearson's correlation coefficient and Student $t$ test. A p $<0.05$ was considered significant.

\section{RESULTS}

The available imaging planes and the anatomic validation in two-dimensional echocardiography: All particular pulsed Doppler velocity patterns of the ligated pulmonary vein were recognized as a continuous or high velocity wave form (Fig. 2). To maintain its consistency with standards that have been established previously, the recommendations presented in this report for imaging planes, display conventions, and anatomic nomenclature of pulmonary veins were as similar as possible to those have been described by other authors $[2,6,21]$.

Probe locations: Two-dimensional echocardiograms with color Doppler flow mappings were obtained from all of the five dogs through three acoustic windows. They are right intercostal, left cranial and left caudal intercostal locations. These locations and the respective image planes are 


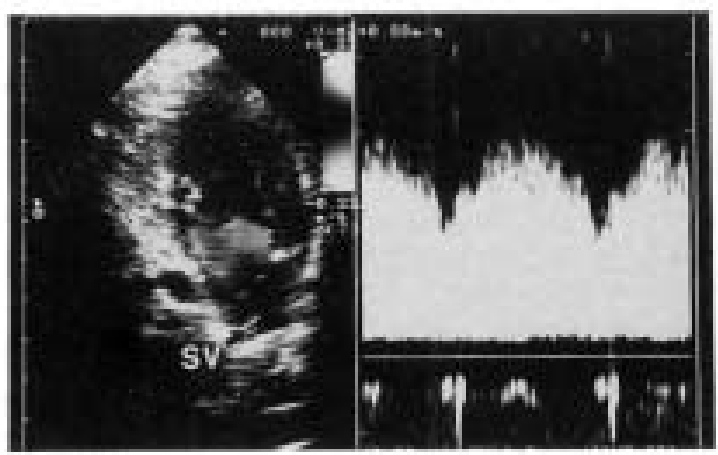

Fig. 2. The pulsed Doppler PVF velocity pattern of right caudal lobe pulmonary vein obtained from left lateral apical 4-chamber view in a dog whose right caudal lobe pulmonary vein was ligated to produce obstruction. Note the continuous and high velocity of the tracings; (SV) sample volume.

described as follows.

Right intercostal location: This acoustic window was located at the area from fourth to fifth intercostal spaces within 3-5 cm of the sternal edge. From this area, a longaxis 4-chamber and a short-axis imaging planes were consistently obtained, in which the pulmonary veins were found. To obtain the long-axis 4-chamber view, the beam plane was oriented parallel to the dog's long axis and then rotated slightly counterclockwise with directing index mark craniodorsally. By directing the beam plane caudally and medially, the long-axis 4-chamber view was obtained. In this view, the color signal of left caudal lobe PVF was observed clearly from about $1 \mathrm{~cm}$ distal to left atrium (Fig. 3-A).

By further rotating the beam plane counterclockwise to make the perpendicular to the long-axis 4-chamber view, a short-axis view could be obtained. With moving the probe toward the heart base slightly, a view between mitral valve level and aortic valve level was obtained (Fig. 3-B). In this view, the left caudal lobe PVF was observed clearly. The direction of index mark in this imaging plane was different from the method described previously, with the index mark pointing to caudodorsally, because rotating the probe counterclockwise was easier.

Left cranial intercostal location: This acoustic window was located at the area from third to fourth intercostal spaces. From this area, a short-axis view was obtained according to the method described by Hagio [6], in which the aortic root was encircled by the right ventricle and the pulmonary artery clockwise. By directing the beam plane caudally from this view slightly and with the beam plane located just beneath the main pulmonary artery, a modified short-axis view was obtained (Fig. 3-C). In this imaging plane, the aortic root was encircled by the right atrium, right ventricle, left auricle and atrium clockwise. The signal of right caudal lobe PVF was observed from about $1.5 \mathrm{~cm}$ distal to left atrium.

Left caudal intercostal location: This acoustic window was located at the area from fifth to sixth intercostal spaces within $3-4 \mathrm{~cm}$ of the sternal edge. From this echo window, three imaging planes were obtained. By orienting the beam plane perpendicularly to the long axis of the body and slightly rotating the probe clockwise, an apical 4-chamber view of the heart was obtained. In this imaging view the right and left caudal lobe PVFs were observed (Fig. 3-D).

The second imaging plane obtained from this echo window was a long-axis 2-chamber view including the left ventricle, mitral valve and left atrium with left auricle (Fig. 3-E). This long-axis 2-chamber view was obtained with orienting the beam plane perpendicularly to the long axis of the body and slightly rotating the probe counterclockwise. The color Doppler signal of the right caudal lobe PVF was seen, but it was short.

The last imaging plane obtained from left caudal intercostal space was a long-axis left ventricular outflow view (Fig. 3-F). With orienting the beam plane nearly parallel to the long axis of the body, then directing the beam plane slightly toward the right lateral of the dog, this view was obtained. The color Doppler signal of the right caudal lobe PVF runs from about $1 \mathrm{~cm}$ distal to left atrium.

The velocity characters of PVF: The PVF wave form demonstrated two forward waves in 19 dogs (95\%), where there was one peak occurring during ventricular systole and another during ventricular diastole (Fig. 4-A). A reversed flow was seen during atrial systole in $11 \operatorname{dogs}(55 \%)$ but not observed in the remaining 9 dogs. In one dog, which had a faster heart rate (198 beats per min), the $S$ wave blended into the preceding $\mathrm{D}$ wave to form a single forward flow wave (Fig. 4-B). Therefore, variables of PVF were analyzed in the rest of the $19 \mathrm{dog}$ shich had adequate recording of $\mathrm{S}$ wave and $\mathrm{D}$ wave.

The parameters of PVF are summarized in Table 1. The mean peak velocity and the VTI of D wave were significantly greater than those of $S$ wave $(p<0.0001)$, with a systolic fraction of $39 \pm 5 \%$. The deceleration time of $D$ wave shortened significantly while the heart rate increasing $(\mathrm{r}=-0.87, \mathrm{p}<0.0001)$ (Fig. 5).

The parameters of TMF are shown in Table 2. There were no significant correlations between the velocities or velocity-time integrals of PVF and TMF directly, whereas the $\mathrm{S}$ wave/D wave VTI ratio correlated significantly with $\mathrm{A}$ wave/E wave velocity ratio and $\mathrm{A}$ wave/E wave VTI ratio $(\mathrm{r}=0.73, \mathrm{p}<0.001$ and $\mathrm{r}=0.72, \mathrm{p}<0.001$, respectively) (Fig. 6). The systolic fraction also correlated with A wave/ $\mathrm{E}$ wave velocity ratio and $\mathrm{A}$ wave/E wave VTI ratio significantly $(\mathrm{r}=0.73, \mathrm{p}<0.001$ and $\mathrm{r}=0.72, \mathrm{p}<0.001$, respectively) (Fig. 6). The mean deceleration time of $D$ wave was longer than the mean deceleration time of $\mathrm{E}$ wave significantly $(\mathrm{p}<0.0001)$.

\section{DISCUSSION}

In the present study, six available imaging planes that enable the observation of PVF with two-dimensional or pulsed Doppler echocardiography were obtained. In the left 


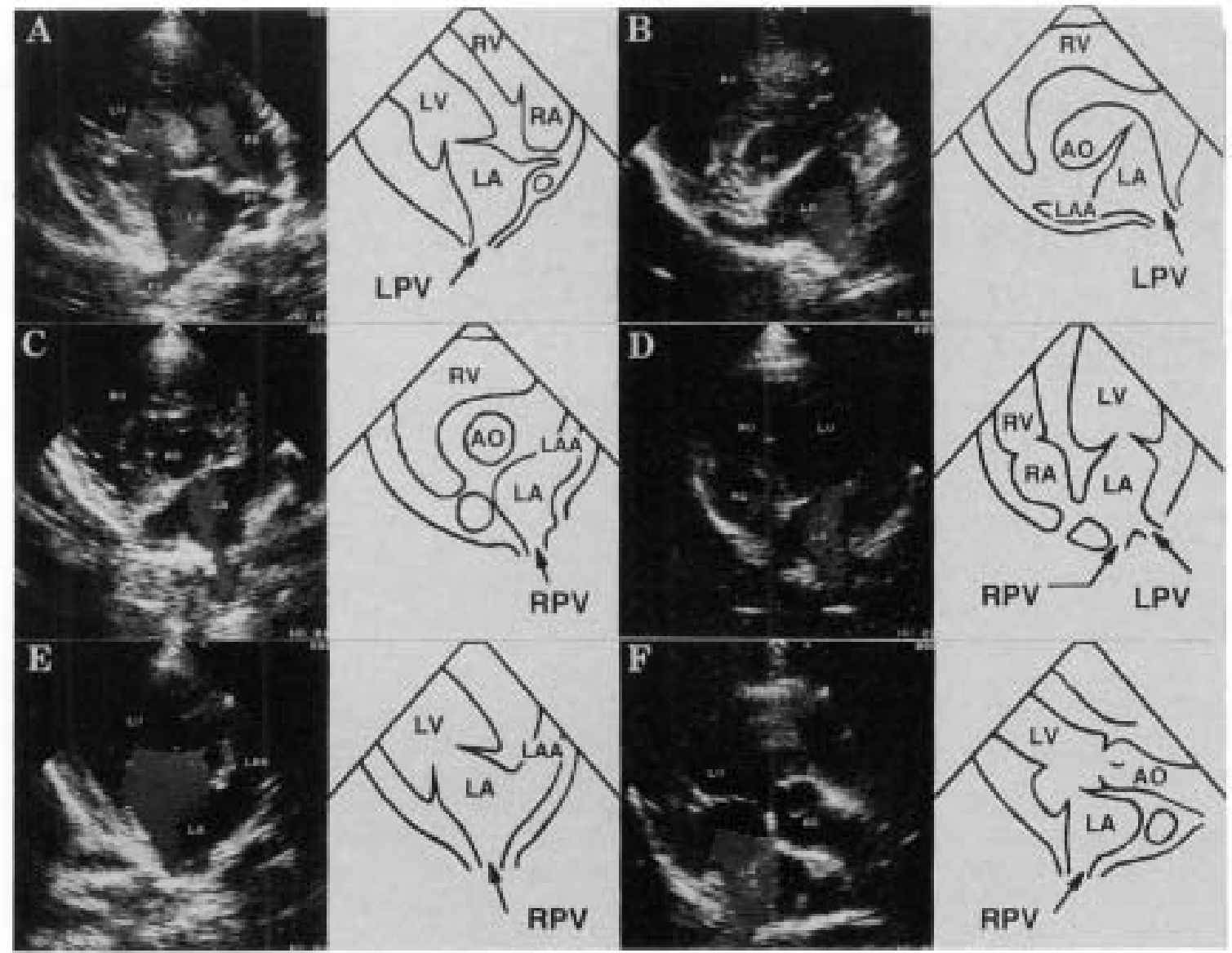

Fig. 3. The available imaging planes for observing pulmonary venous flow: A. right lateral long-axis 4-chamber view; B. right lateral short-axis view; C. left lateral cranial short-axis view; D. left lateral caudal apical 4-chamber view; E. left lateral caudal long-axis 2-chamber view; F. left lateral caudal long-axis left ventricular outflow view. (LA) left atrium; (LV) left ventricle; (LAA) left auricle; (RA) right atrium; (RV) right ventricle; (RPV) right caudal lobe pulmonary vein; (LPV) left caudal lobe pulmonary vein.

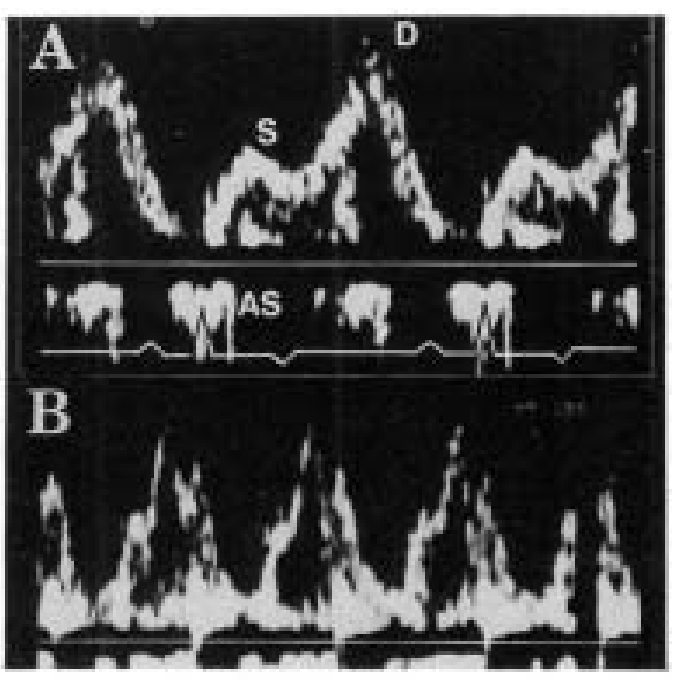

Fig. 4. A. The normal velocity pattern of pulmonary venous flow in a dog. B. In one dog which had a fast heart rate (198 beats per min), the $\mathrm{S}$ wave blends into the preceding $\mathrm{D}$ wave to form a single forward flow wave. lateral apical 4-chamber view, the right and left caudal lobe pulmonary veins were observed simultaneously and clearly, and the TMF could be evaluated in the same imaging plane. However, the angle between left caudal lobe PVF and Doppler sampling line was usually larger than 20 degrees. On the other hand, the right caudal lobe PVF was almost in parallel with the Doppler sampling line. Therefore, authors considered that this imaging plane could be applied as a standard view for observing the PVF of the right caudal lobe pulmonary vein in dogs. In the left lateral short-axis view, the right pulmonary venous color signal was imaged longer than that could be observed in other imaging planes. Unfortunately, this imaging plane was performed with some difficulty, because the obliquity between the probe and surface of body was large, which made the rigorous contact difficult. In the right lateral long-axis 4-chamber view, the left caudal lobe pulmonary vein was observed clearly with a small angle between the Doppler sampling line and the blood flow. Accordingly, we considered that the right lateral longaxis 4-chamber view could be applied for examining PVF with M-mode echocardiography simultaneously at need, 
Table 1. Parameters obtained from pulmonary venous flow velocity pattern in 19 healthy dogs

\begin{tabular}{lccc}
\hline & mean \pm SD & Range & N \\
\hline $\begin{array}{l}\text { Peak velocity of systolic flow } \\
\text { (S) (cm/s) }\end{array}$ & $31.13 \pm 4.92$ & 21.37 to 39.04 & 19 \\
$\begin{array}{l}\text { Peak velocity of diastolic flow } \\
\text { (D) (cm/s) }\end{array}$ & $46.49 \pm 6.79$ & 33.73 to 57.75 & 19 \\
S/D ratio & $0.67 \pm 0.09$ & 0.53 to 0.81 & 19 \\
Peak velocity at atrial & $23.33 \pm 4.58$ & 17.87 to 31.17 & 11 \\
$\quad$ contraction (AS) (cm/s) & & & \\
VTI of systolic flow (VTIs) (cm) & $5.14 \pm 0.82$ & 4 to 7.33 & 19 \\
VTI of diastolic flow (VTId) (cm) & $8.18 \pm 1.84$ & 5.67 to 12 & 19 \\
VTIs/VTId ratio & $0.65 \pm 0.14$ & 0.42 to 0.90 & 19 \\
Systolic fraction & $0.39 \pm 0.05$ & 0.30 to 0.47 & 19 \\
(VTIs/VTIs+VTId) & & & \\
Deceleration time (DTd) (ms) & $108.70 \pm 14.06$ & 82.73 to 133.40 & 19 \\
Heart rate & $120 \pm 19$ & 86 to 148 & 19 \\
\hline
\end{tabular}

VTI=velocity-time integral: s=systole; $d=$ diastole;

Table 2. Parameters obtained from transmitral flow velocity pattern in 19 healthy dogs

\begin{tabular}{lcc}
\hline & mean \pm SD & Range \\
\hline $\begin{array}{l}\text { Peak velocity of early diastolic } \\
\text { fillign flow (E) (cm/s) }\end{array}$ & $65.07 \pm 11.16$ & 47.63 to 82.90 \\
$\begin{array}{l}\text { Peak velocity of late diastolic } \\
\text { filling flow (A) (cm/s) }\end{array}$ & $40.73 \pm 6.46$ & 31.66 to 50.83 \\
A/E ratio & $0.63 \pm 0.08$ & 0.49 to 0.77 \\
VTI of early diastolic filling & $6.02 \pm 1.11$ & 4.33 to 8.33 \\
flow (VTIe) (cm) & $2.53 \pm 0.41$ & 2 to 3.33 \\
VTI of late diastolic filling & & \\
flow (VTIa) (cm) & $0.43 \pm 0.06$ & 0.32 to 0.54 \\
VTIa/VTIe ratio & $88.69 \pm 10.77$ & 65.13 to 100 \\
Decleration time (DTe) $(\mathrm{ms})$ & $109 \pm 20$ & 66 to 141 \\
Heart rate &
\end{tabular}

VTI=velocity-time integral; e=early filling flow; a=late filling flow

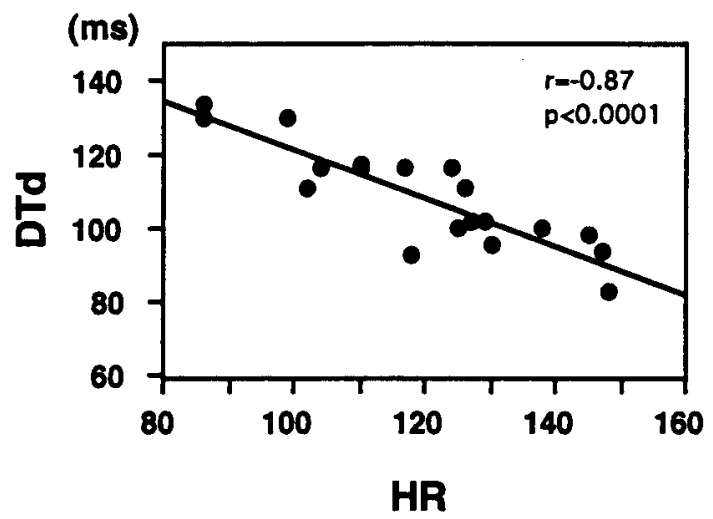

Fig. 5. The deceleration time of pulmonary venous D wave (DTd) shortens with heart rate (HR) increases significantly.

because the M-mode echocardiography is performed from right lateral thoracic wall preferably. In this study, except the right and left caudal lobe pulmonary veins, no available
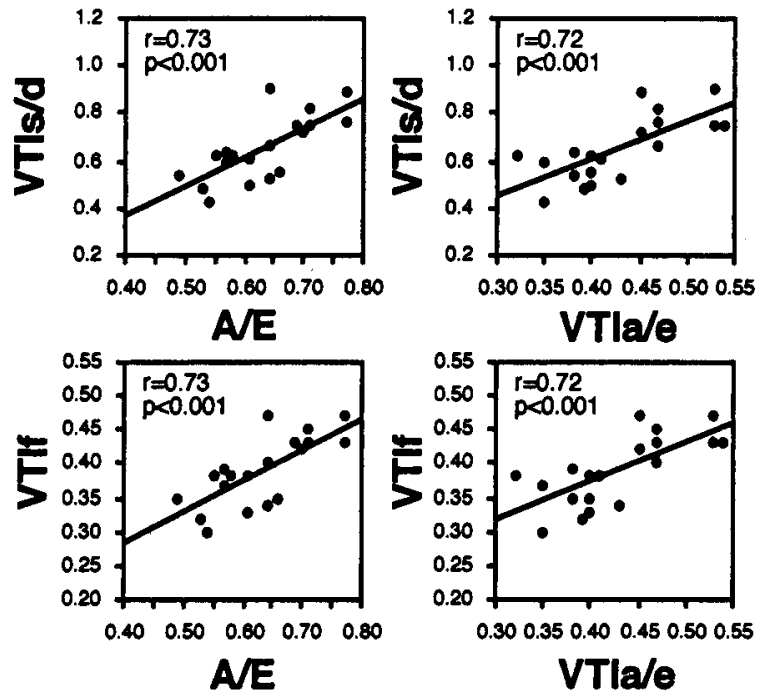

Fig. 6. The pulmonary venous VTI ratio of S wave to D wave (VTIs/d) correlates significantly with transmitral velocity ratio of $\mathrm{A}$ wave to $\mathrm{E}$ wave $(\mathrm{A} / \mathrm{E})$ and $\mathrm{VTI}$ ratio of $\mathrm{A}$ wave to E wave (VTIa/e)(top panel). The correlations between the pulmonary venous systolic fraction (VTIf) and A/E or VTIa/e are shown at bottom panel.

image was obtained with two-dimensional or Doppler echocardiography to observe other pulmonary veins. On the other hand, with color Doppler flow mapping it is easy to certify the orifice of pulmonary vein, and to make the Doppler sampling line parallel to the blood flow of pulmonary vein.

In the present study, the PVF wave form demonstrated biphasic forward flow during systole and diastole in 19 of 20 dogs examined, which are consistent with the previous reports in human or experimental dog model [1, 3, 9, 10, $17,19,20]$. In human studies, $30-37 \%$ of the $S$ wave were further divided into two waves with the former occurring during atrial diastole $[10,17]$. However, the biphasic S 
wave was not observed in this study. In present study, the sweep speed was set at $1.25 \mathrm{sec} / \mathrm{screen}$, which equals 496 pixels/sweep. In this setting, the resolving power is about $2.5 \mathrm{msec} / \mathrm{pixel}$. As the result, the motion of a subject faster than $2.5 \mathrm{msec}$ was not recorded on the screen. It might be a reason we could not observe the biphasic $S$ wave. The reversed flow at atrial contraction was observed in 11 of the 20 dogs $(55 \%)$, which was lower than the detection rate of atrial systolic reversed flow in human with transthoracic echocardiography [10]. In one dog, which had a fast heart rate (198 beats per min), the $S$ wave blended into the preceding $\mathrm{D}$ wave to form a single forward flow wave. It is unknown what heart rate would discriminate the $S$ wave and $\mathrm{D}$ wave. However, it was reported previously that the two peaks of TMF became fused and were no longer separable when the heart rate $>200$ in dogs [8]. Smallhorn et al. [17] proposed that increased heart rate affects the flow pattern by shortening the period of diastole such that blending the systolic and diastolic peaks occur until only one peak is observed at a rapid rate. As moving with heart beat or respiration, wall motion artifacts may interfere with the obtaining of the atrial reversed flow velocity, and the artifacts will disturb the quality of spectrum of PVF, especially during left atrium contract and left ventricle systole.

The $\mathrm{S}$ wave occurs as the mitral annulus descends in a direction toward the apex and the atrial relaxes [7]. During this duration, the atrium acts as a reservoir to receive the blood filling from the pulmonary veins. The D wave occurs after the opening of mitral leaflet while the left atrial pressure decreases. During this phase, the left atrium acts as an open conduit to transfuse blood from the pulmonary vein through the mitral valve into the left ventricle. During atrial contraction, blood flow transfuses through the mitral valve and produces a small reverse flow into the pulmonary veins. In patients with abnormal myocardial relaxation, e.g., hypertrophic cardiomyopathy or coronary artery disease, the TMF velocity pattern usually appears a lower $\mathrm{E}$ wave and a compensatory higher A wave. Furthermore, as the enddiastolic filling pressure increases gradually, the compliance of left ventricle will reduce significantly. As the result, the velocity decreases during left atrium contraction to produce a "pseudonormalization" of the abnormal TMF pattern, i.e., the A/E velocity ratio $<1[9,15]$. This pseudonormalization will make the evaluation of diastolic function difficult and complex. On the other hand, the reverse flow in the pulmonary vein will become more prominent than the A wave of TMF by afterload mismatch when left ventricle compliance decreased and the left atrial pressure increased [9]. The predominant reversed flow in pulmonary vein could be used to distinguish the pseudonormalization from normal left ventricular filling. Therefore, the diastolic function of heart can be diagnosed more accurately by analyzing the TMF simultaneously with PVF even when the pseudonormalization appears [15].

In this study, the PVF wave forms of normal dogs were similar to those of sedated or anesthetized dog models $[1,3$,
19]. Appleton et al. [3] reported that pulmonary venous diastolic velocity was higher than systolic velocity $(66 \pm 9$ versus $36 \pm 11 \mathrm{~cm} / \mathrm{s}$ ) with a systolic fraction of $31 \pm 8 \%$ in lightly sedated dogs with transthoracic pulsed Doppler echocardiography. However, they did not discuss the reversed flow at atrial contraction. Akita et al. [1] proposed that a forward PVF flow rather than a reversed flow occurs during left atrial contraction, similar to Rajagopalan et al. [16] reported. Conversely, in the present study the reversed flow at atrial contraction was observed in 11 of 20 dogs, and no forward flow at atrial contraction was observed. It was considered that the difference might be attributed to the sampling methods. In our experiment, the blood flow at the orifice of pulmonary vein was measured by pulsed Doppler ultrasound, but Akita and Rajagopalan used trans-time ultrasonic flowmeter and cuff electromagnetic flow probe, respectively, to measure the blood flow. The reversed flow of atrial contraction in normal dogs may be too small to record by the flowmeter probe.

In the present study, there was no direct correlation obtained among the velocity or VTI of PVF and those of TMF during systole or diastole. However, the VTI ratio of $\mathrm{S}$ wave to $\mathrm{D}$ wave and the systolic fraction correlated significantly with the velocity ratio and VTI ratio of A wave to $\mathrm{E}$ wave. The various sizes of heart in dogs may influence the angle between the Doppler sampling line and the PVF, which could affect the recording of velocity. Therefore, analyzing the ratio of velocity or VTI simultaneously was considered to be more important than evaluating the velocity only in dogs.

It should be considered that several physiologic factors such as heart rate, respiratory cycle or age will influence the PVF filling $[9,10,17,20]$. In this study, the deceleration time of $\mathrm{D}$ wave shortened significantly with the heart rate increase. One dog had sinus tachycardia (198 beats per min) to produce blending of $\mathrm{S}$ wave and $\mathrm{D}$ wave. Therefore, the heart rate must be taken into considered when Doppler measurements of PVF are obtained.

The unpublished data of our laboratory shows the velocity of PVF during inspiration was significantly lower than during expiration in anesthetized dogs, and the wave form during expiration was stable enough for consecutive recording of several cardiac cycles. However, few certain changes of the wave form could be related to the influence of respiration in present study. Klein and Tajik [9] reported that spontaneous respiratory cycle has little effect $(<4 \%)$ on human PVF.

The senescent process may affect PVF as well as TMF. In this study, we used relatively younger $\operatorname{dog}$ s for investigation, and obtained the results that the $S$ wave/D wave velocity and VTI ratios were lower than 1 . However, in human studies $[9,10]$, the D wave of PVF was higher than the $S$ wave in young subjects, while the $S$ wave was higher than the $\mathrm{D}$ wave in subjects who were older than 50 years old. Although it is not clearly known whether those ratios will change with aging in the dogs, the age might be an important factor when interpret the data from PVF. 
In conclusion, the results of this study are: (1) at least, six echocardiographic imaging planes enable us to observe the right or left caudal lobe PVF. The left lateral apical 4chamber view is the best view for assessing the right caudal lobe PVF. (2) The PVF consists of two or three phases, i.e., systolic and diastolic forward flow, and sometimes a reverse flow at atrial contraction. The peak velocity and VTI of diastolic forward flow are higher and larger than systolic forward flow in normal, relatively young conscious dogs. Thus, observation of pulmonary venous flow in dogs with transthoracic two-dimensional or Doppler echocardiography is feasible, and the results of the normal dogs can used for the comparison with pulmonary venous flow patterns in disease states.

\section{REFERENCES}

1. Akita, S., Ohte, N., Hashimoto, T., Kobayashi, K. and Narita, H. 1995. Effects of volume loading on pulmonary venous flow pattern in dogs with normal left ventricular function. Angiology 46: 393-399.

2. Anderson, W. D. and Anderson, B. G. 1994. Part 3. Thorax: The Lungs. pp. 473-488. In: Atlas of Canine Anatomy. Lea \& Febiger, Philadelphia.

3. Appleton, C. P., Gonzalez, M. S. and Basnight, M. A. 1994. Relationship of left atrial pressure and pulmonary venous flow velocities: Importance of baseline mitral and pulmonary venous flow velocity patterns studied in lightly sedated dogs. J. Am. Echocardiogr. 7: 264-275.

4. Guntheroth, W. G., Gould, R., Butler, J. and Kinnen, E. 1974. Pulsatile flow in pulmonary artery, capillary, and vein in the dog. Cardiovasc. Res. 8: 330-337.

5. Habel, R. E. 1978. Lesson 1. Teeth. pp. 15-17. In: Applied Veterinary Anatomy, 2nd ed., Edwards Brothers Incorporated, Michigan.

6. Hagio, M. 1985. Two-dimensional echocardiography in the dog: Technique and clinical utility. Adv. Anim. Cardiol. 18: 7-21 (in Japanese).

7. Keren, G., Sonnenblick, E. H. and LeJemtel, T. H. 1988. Mitral anulus motion: Relation to pulmonary venous and transmitral flows in normal subjects and in patients with dilated cardiomyopathy. Circulation 78: 621-629.

8. Kirberger, R. M., Bland-van den Berg, P. and Darazs, B. 1992. Doppler echocardiography in the normal dog: Part I Velocity findings and flow patterns. Vet. Radiol. Ultrasound. 33: 370-379.

9. Klein, A. L. and Tajik, A. J. 1991. Doppler assessment of pulmonary venous flow in healthy subjects and in patients with heart disease. J. Am. Soc. Echocardiogr. 4: 379-392.
10. Masuyama, T., Lee, J. M., Tamai, M., Tanouchi, J., Kitabatake, A. and Kamada, T. 1991. Pulmonary venous flow velocity pattern as assessed with transthoracic pulsed Doppler echocardiography in subjects without cardiac disease. Am. J. Cardiol. 67: 1396-1404.

11. Morgan, B. C., Abel, F. L., Mullins, G. L. and Guntheroth, W. G. 1966. Flow patterns in cavae, pulmonary artery, pulmonary vein, and aorta in intact dogs. Am. J. Physiol. 210: 903-909.

12. Morgan, B. C., Dillard, D. H. and Guntheroth, W. G. 1966. Effect of cardiac and respiratory cycle on pulmonary vein flow, pressure, and diameter. J. Appl. Physiol. 21: 12761280.

13. Morkin, E., Collins, J. A., Goldman, H. S. and Fishman, A. P. 1965. Pattern of blood flow in the pulmonary veins of the dog. J. Appl. Physiol. 20: 1118-1128.

14. Ogawa, S., Oki, T., Iuchi, A., Hosoi, K., Fujimoto, T., Kiyoshige, K., Tabata, T., Tanimoto, M., Fukuda, K. and Fukuda, N. 1993. Evaluation of pulmonary venous flow patterns in left heart failure: A study using transesophageal Doppler echocardiography. J. Cardiol. 23: 193-204 (in Japanese).

15. Pai, R. G. and Buech, G. C. 1996. Newer Doppler measures of left ventricular diastolic function. Clin. Cardiol. 19: 277 288.

16. Rajagopalan, B., Friend, J. A., Stallard, T. and Lee, G de J. 1979. Blood flow in pulmonary veins: I Studies in dog and man. Cardiovasc. Res. 13: 667-676.

17. Smallhorn, J. F., Freedom, R. M. and Olley, P. M. 1987. Pulsed Doppler echocardiographic assessment of extraparenchymal pulmonary vein flow. J. Am. Coll. Cardiol. 9: 573-579.

18. Tabata, T., Oki, T., Fukuda, N., Iuchi, A., Kawano, T., Manabe, K., Tanimoto, M., Kageji, Y., Sasaki, M., Hama, M. and Ito, S. 1996. Transesophageal pulsed Doppler echocardiographic study of pulmonary venous flow in mitral stenosis. Cardiology 87: 112-118.

19. Takaki, A., Katayama, K., Sakai, H., Tanaka, N. and Matsuzaki, M. 1996. Assessment of pulmonary venous and transmitral flow in closed-chest dogs under various loading conditions by transesophageal Doppler echocardiography. Jpn. Circ. J. 60: 115-123.

20. Tei, C., Yutsudo, T., Shibata, K., Shah, P., Park, J. C., Horikiri, Y., Minakami, N., Sotoyama, Y. and Tanaka, N. 1991. Pulmonary venous flow patterns in normal subjects and cardiac patients: A transesophageal echocardiographic study. $J$. Cardiol. 21 (Suppl. XXVI): 91-102 (in Japanese).

21. Thomas, W. P. 1984. Two-dimensional, real-time echocardiography in the dog. Technique and anatomic validation. Vet. Radiol. 25: 50-64. 